ISSN 1669-2438

Revista de Psicología. Año 2020. Vol. 16, № 32, pp. 79-103

ISSN-electrónico 2469-2050

\title{
Las relaciones entre juego dramático y autorregulación: Una revisión sistemática
}

Relationship between sociodramatic play and self-regulation: A systemic revision

Mercedes Estrugo*

Karen Moreira**

\section{Resumen}

El objetivo del presente trabajo es realizar una revisión bibliográfica que permita analizar en qué medida el juego dramático se relaciona con el desarrollo de la autorregulación. Para esto, se consideró la franja etaria que va de los tres a los seis años, franja en la que se produce un desarrollo notable del juego y de la autorregulación. Para el análisis de ésta relación, se realizaron una serie de búsquedas en el Portal Timbó que dieron como resultado final 7 artículos de investigaciones empíricas escritos entre el año 2002 y el 2020. Como resultado de esta revisión se podría decir que el juego dramático maduro está fuertemente relacionado con la adquisición de las habilidades de autorregulación que se desarrollan en esta etapa de la vida y son fundamentales para el paso al ciclo de educación primaria, y para toda la vida posterior. Dos elementos muy importantes por considerar son la calidad del juego y la inclusión de elementos de fantasía en ellos.
Palabras clave: juego dramático, autorregulación, funciones ejecutivas, etapa preescolar

\section{Abstract}

In this article, we review studies that analyze the relationship between sociodramatic play and self-regulation in 3-6 years old children. It is well established that Early childhood is a landmark period for laying the foundations of self-regulation. We searched the Portal Timbó database using combinations of the keywords. We included reports that were published in English and specifically examined how Sociodramatic play promotes Self-regulation development. Seven published articles between 2002-2020 met our selection criteria. Although few studies explored the relationship between sociodramatic play and self-regulation, they evidence that mature sociodramatic play is

*Universidad de la República, Uruguay. Mail de contacto: mercedesestrugo@hotmail.com **Universidad de la República, Uruguay. Mail de Contacto: moreirak@psico.edu.uy DOI: https://doi.org/10.46553/RPSI.16.32.2020.p79-103

Fecha de recepción: 7 de mayo de 2020 - Fecha de Aceptación: 25 de septiembre de 2020 
strongly related with self-regulatory skill acquisition developed at a pre-school stage. Two very important elements to consider are quality in play and the inclusion of fantasy elements within them.

Keywords: pretend play, self-regulation, executive function, preschool

\section{Introducción}

Dentro de la perspectiva histórico- cultural, se ha señalado la existencia de una conexión causal entre el juego dramático y el desarrollo de las funciones ejecutivas en la etapa preescolar. Este trabajo se propone revisar esta literatura partiendo de una caracterización de la edad preescolar, de una definición amplia de los procesos ejecutivos y de autorregulación (AR), así como también de los cambios que se dan en las relaciones sociales en este período, y que afectan el desarrollo de la AR.

\section{Caracterización de la etapa preescolar}

La edad preescolar, abarca de los tres a los seis años aproximadamente, y queda definida por la aparición de la imaginación como función psicológica central, que le permite al niño mediar entre los deseos que no puede satisfacer en forma inmediata y las demandas de la realidad (Vygotsky, 1996). La imaginación se pone en evidencia en la capacidad del niño de jugar a "como si", y de mantener la separación entre ficción y realidad (Carlson, White \& Davis-Unger, 2014; Nelson, 2007). En este momento el lenguaje comienza a cumplir una función discursiva, de modo que los niños se vuelven capaces de reconstruir lingüísticamente $\mathrm{y}$ narrar sus experiencias, lo que organiza verbalmente su pensamiento y desarrolla la memoria autobiográfica (Bruner, 1991; Nelson, 2007). La interiorización del lenguaje y su uso como estructurador del pensamiento y como herramienta que guía la conducta representa uno de los logros más significativos para el desarrollo cognitivo en esta etapa de la vida (Elias \& Berk, 2002). En este período el niño comienza a percibirse como agente de actos voluntarios, toma decisiones y reclama ser consultado por todo aquello que lo implica (Vygotsky, 1996, 2000). La imagen que tienen del niño los adultos de su entorno cambia drásticamente y con ello se producen cambios en las relaciones sociales del niño. En palabras de Zaporozhets (1980) "la infancia preescolar es el período de más intenso desarrollo físico y mental de la personalidad humana" (p. 69). Éste es un período de gran plasticidad neural y permite influenciar en el desarrollo mucho más profundamente que en períodos posteriores. A pesar de que siempre se puede intervenir en el desarrollo, cuando estas intervenciones se hacen a destiempo se requiere de mayor esfuerzo para lograr las modificaciones deseadas (Lipina \& Segretín, 2015).

\section{Relaciones sociales y juego}

A medida que van interiorizando formas culturales de conducta, los niños reconstruyen su propia actividad psicológica y llegan a apropiarse de los recursos que le brinda el mundo cultural en el que viven. Estos recursos refieren tanto a información necesaria para integrar determinada cultura como a cierta forma de funcionamiento psicológico. Todo lo que los niños aprenden les sirve de guía para interpretar el mundo y la 
diversidad de fenómenos sociales en los que se pueden ver involucrados (Nicolopoulou, 1993). Debido a esto se afirma que las funciones psicológicas superiores son un producto derivado de las relaciones sociales, a partir de las cuales emergen (Vygotski, 1997; Wertsch, 1999). En este sentido es conocida la ley genética del desarrollo formulada por Vygotski sobre que:

Toda función en el desarrollo cultural del niño aparece dos veces, en dos planos; primero en el plano social y después en el plano psicológico, al principio entre los hombres como categoría interpsíquica y luego en el interior del niño como categoría intrapsíquica. Lo dicho se refiere por igual a la atención voluntaria, a la memoria lógica, a la formación de conceptos y al desarrollo de la voluntad. (Vygotski, 1995, p. 150)

En el proceso de interiorización de las relaciones sociales el juego cumple un papel clave, pues en el juego el niño imita a las personas de su entorno. Esto le posibilita ir creando situaciones novedosas que va compartiendo y explorando (Karpov, 2005). Esto justifica la importancia que los sistemas educativos asignan al juego en la etapa preescolar.

\section{EI "juego dramático" como práctica cultural y social}

El juego es la actividad rectora en la edad preescolar (Elkonin, 1980; Vygotski, 2000), es decir, la actividad alrededor de la cual se organiza el funcionamiento psicológico en una determinada fase del desarrollo. El juego que nos interesa tratar aquí (juego dramático), surge en la etapa preescolar cuando el niño se vuelve capaz de separar el campo visual directo del campo de sentido (Vygotski, 1996), cuando comienza a actuar con independencia de lo que ve, decide a qué desea jugar y utiliza los elementos de su entorno para apoyar el juego. Lo novedoso de este momento del desarrollo es que puede utilizar un objeto para representar otro, aunque el objeto real no tenga ningún parecido con lo que representa o incluso, en una etapa posterior, puede imaginar el escenario sin necesitar valerse de objetos (Hakkarainen \& Bredikyte, 2015; Nelson, 2007; Vygotsky, 2005).

Este tipo de juego nos permite identificar en la acción del niño una situación imaginaria, roles definidos y reglas asociadas a estos roles (Hakkarainen \& Bredykite, 2015; Vygotski, 1996). Este juego puede verse como una actividad social, incluso cuando un niño juega solo, ya que en el tema o las situaciones de su juego está expresando elementos socioculturales (Elkonin, 1980; Nicolopoulou, 1993).

El juego dramático presenta un desarrollo evolutivo con cuatro grandes fases (Elkonin, 1980). Las dos primeras fases -que corresponden a un juego inmaduro- tienen a los objetos como centro. Aquí son los objetos y no la voluntad del niño los que guían su juego, la interacción entre los participantes es limitada, los roles son estereotipados y están pobremente representados. Un ejemplo típico de estas fases lo encontramos cuando un niño juega a los papás porque dispone de una muñeca, su acción está gobernada por el objeto, más que por un plan interno. Las dos últimas fases -que corresponden al juego 
maduro- se centran en la adopción y ejercicio de roles sociales. En este momento, el juego tiene una orientación social, los roles se negocian y se actúan durante un período de tiempo prolongado, convirtiéndose el niño en el personaje que representa. Aquí los niños pueden, progresivamente, distanciarse de su yo y adoptar la perspectiva del rol que representan. En esta línea Bodrova \& Leong (2004), mencionan como características principales del juego maduro:

1. Temas complejos entrelazados, los niños pueden integrar diversos temas en la situación imaginaria, también pueden incorporar nuevas personas e ideas generando situaciones más complejas y escenarios más ricos, muchas veces los niños pasan más tiempo planificando el juego que representando lo que planificaron.

2. Roles complejos entrelazados, bien diferenciados y articulados, lo que además implica que los niños asumen y coordinan varios roles simultáneamente.

3. Representaciones y acciones simbólicas, donde los niños pueden representar lo que desean o necesitan con objetos o sin ellos; en cierto punto pueden representar objetos con gestos y asignar roles a los objetos.

4. El marco temporal extendido, los niños representan en su juego períodos de tiempo extensos, se vuelven capaces de retomar el guión del juego en caso de interrupciones.

5. Extenso uso del lenguaje para describir el escenario, los roles y las acciones a seguir.

6. Construcción escenarios complejos que pueden cambiar de acuerdo a los deseos de los jugadores, que se coordinan, y que incluyen temas no necesariamente cotidianos, pudiendo incorporar elementos de la fantasía provenientes de cuentos u otras fuentes.
Los patrones maduros de juego dramático se desarrollan a través de interacciones sociales. Tal como sucede con otros aspectos del desarrollo, la adquisición de un tipo de juego maduro no se da automáticamente a medida que los niños crecen. El niño debe aprender a jugary quienes ofrecen asistencia en este aprendizaje son los adultos -padres y maestros- y sus pares con mayor experiencia (Bodrova, Germeroth \& Leong, 2013; Bredekamp, 2005). En este sentido el juego como un fenómeno históricocultural es dependiente del grado y la calidad de la mediación del adulto (Bodrova, 2008; Karpov, 2005). Como se mencionó con anterioridad, el juego es la actividad rectora en la edad preescolar, la actividad en la que se expresan los máximos niveles de desarrollo, y por ello, debemos comprender su contribución específica al desarrollo cognitivo y social. Entender la dinámica de desarrollo del juego permitirá identificar estrategias de intervención para potenciarlo. Entre estas estrategias puede mencionarse el involucramiento en situaciones imaginarias con roles, reglas e interacciones lingüísticas prolongadas (juego dramático maduro) (Bodrova \& Leong, 2004; Hakkarainen \& Bredikyte, 2015).

\section{La autorregulación y su desarrollo en la edad preescolar}

Se define a la autorregulación (AR) como la habilidad de manejar el comportamiento, la cognición y la emoción para actuar de forma adaptativa. "Supone la capacidad para superar en forma controlada, tendencias naturales y automáticas, deseos y conductas de cara al logro de objetivos a más largo plazo (...) teniendo en cuenta las 
normas y convenciones sociales." (CanetJuric, Introzzi, Andrés \& Stelzer, 2016, p. 108). La evidencia sugiere que estas habilidades son complejas, comienzan a desarrollarse desde la infancia y en los años preescolares tienen un desarrollo muy importante (Garon, Bryson \& Smith, 2008). Whitebread \& Basilio (2012) plantean que "las primeras formas simples de cada una de las funciones ejecutivas fundamentales pueden observarse en niños muy pequeños, y que hay claros desarrollos durante el período preescolar derivados de los progresos en el control voluntario, es decir, de la creciente capacidad para la autorregulación" (p. 19). En los niños muy pequeños éstas son habilidades altamente dependientes del contexto y con el tiempo el niño las va interiorizando.

Canet-Juric et al. (2016) identifican tres componentes principales en la AR: los estándares o metas individuales, que son los objetivos o metas que se trazan las personas y hacia los que dirigen su comportamiento; el monitoreo, que es la capacidad para notar la distancia entre el estado actual y el que se desea alcanzar; y los recursos, que son los procesos responsables de generar el cambio necesario para alcanzar el objetivo deseado. "Estos recursos son los que permiten vencer las tentaciones y limitaciones que las personas encuentran en el camino y que obstaculizan el logro de objetivos." (CanetJuric et al., 2016, p. 111). Gran parte de estos recursos son las funciones ejecutivas (FE).

En algunas ocasiones los conceptos de AR y FE pueden considerarse como sinónimos, "cuando las FE son empleadas al servicio de la AR, entonces FE y AR son prácticamente lo mismo" (Nigg, 2016, p. 8). Sin embargo, Nigg (2016) plantea que las FE abarcan un espectro más amplio de acciones. Las FE son un grupo de capacidades cognitivas que hacen posible que la $\mathrm{AR}$ suceda, pero también se utilizan en otro tipo de procesos -como por ej. en la resolución de problemas matemáticos- donde la $\mathrm{AR}$ no es necesaria.

Las FE han sido definidas de diferentes maneras, recurriendo a diferente terminología, y en el marco de diferentes teorías (Bodrova, Leong \& Akhutina, 2011; Diamond, 2013; Jurado \& Rosselli, 2007; Miyake, 2000). En la teoría histórico-cultural el concepto de AR y FE coinciden, pues la AR es entendida como el dominio de la propia conducta a través de medios culturales, lo que permite que el funcionamiento psicológico se organice sobre la base de planes autodirigidos, más que de la respuesta a la estimulación del entorno inmediato (Bodrova et al., 2011). Estos enfoques sostienen además que el origen de los procesos autorregulatorios está en las relaciones sociales de las que el niño participa, identificando como génesis de la AR la interiorización de las formas sociales de regulación de la acción (Bernier et. al, 2011; Moriguchi, 2014) y el desarrollo de un lenguaje interior con función de control cognitivo (Díaz, et. al, 1998, Fernyhough, 2010; Zelazo, 2003).

Dentro de la psicología cognitiva el concepto de FE tiene una historia extensa. Miyake et. al (2000) establecen uno de los principales mojones en el campo de estudio proponiendo un modelo tripartito de "unidad en la diversidad" que incluye: el cambio de set mental, el monitoreo y actualización de representaciones en la memoria de trabajo y la inhibición de respuestas prepotentes. Este modelo fue propuesto y analizado con 
población adulta, pero su extrapolación a población infantil no ha sido del todo exitosa. Estudios con niños mostraron que modelos de un único factor, cuyos componentes se diferencian a lo largo del desarrollo, resultó más parsimonioso (Carlson, 2005; Diamond, 2013, 2016; Hughes, 2013).

Diamond $(2013 ; 2016)$ define a las FE como una familia de procesos mentales de arriba-abajo, necesarios cuando la persona debe concentrarse y poner atención para llevar a cabo una tarea o en vías de dar una respuesta más adecuada cuando las respuestas automáticas o instintivas no son apropiadas, son insuficientes o inexistentes. Desde este punto de vista los conceptos de AR y FE coinciden sólo parcialmente, ya que la AR se solapa principalmente con el componente de Control Inhibitorio (Diamond, 2013, 2016). En el modelo de Diamond (2013) las FE se diferencian en tres componentes que trabajan juntos al servicio de la resolución de problemas:

1. El control inhibitorio, que nos permite controlar la atención, el comportamiento, los pensamientos $\mathrm{y} / \mathrm{o}$ las emociones e ignorar fuertes predisposiciones internas o atractivos externos para poder actuar de forma más apropiada. Dentro del control inhibitorio se distinguen (Diamond, 2016): el control de la interferencia (que permite inhibir pensamientos y recuerdos que interfieren con la consecución de nuestras metas), la inhibición de respuestas motoras y la sustitución de respuestas prepotentes por respuestas menos automáticas pero más apropiadas a la situación, así como también la postergación de la gratificación.

2. La memoria de trabajo, que es la capacidad de retener y manipular mentalmente información necesaria para el cumplimiento de una tarea determinada. Incluye un sistema ejecutivo central y dos sistemas esclavos, uno de naturaleza verbal (bucle fonológico) y otro viso-espacial (agenda viso-espacial). La memoria de trabajo está implicada en la resolución de cualquier tarea de resolución no rutinaria (que requiera recombinación creativa de información) (Diamond, 2013; 2016).

3. La flexibilidad cognitiva, que se construye a partir de las dos anteriores y permite cambiar de perspectiva espacial -cómo se vería algo si se viera desde otra direccióno interpersonal -ver las cosas desde el punto de vista del otro-. También involucra la posibilidad de cambiar nuestra manera de pensar tras obtener nueva información, poder admitir cuando nos equivocamos y ser flexibles como para adaptarnos a cambios en las prioridades $o$ en las demandas provenientes del exterior. La flexibilidad aparece más tardíamente que el control inhibitorio y la memoria de trabajo, en una etapa posterior del desarrollo.

$\mathrm{Al}$ hablar de FE también es necesario considerar la motivación y el compromiso del niño con la actividad. Si la complejidad de la información que se le da es demasiado grande o si la actividad provoca demasiado estrés, las FE no funcionan, se ven agobiadas. Si, por el contrario, la actividad es demasiado simple y presentada de forma poco interesante, tampoco se recurre al uso de FE. El desarrollo de las FE depende -además de la maduración biológica- de que se le presenten al niño actividades con un nivel apropiado de complejidad y del soporte que le brinde el medio para poder regular el estrés, siendo ésta la base de la noción vygotskiana de zona de desarrollo próximo (Blair \& Raver, 2015). 
Zelazo, Hongwanishkul, Happaney \& Lee (2005) propusieron distinguir dos aspectos de las FE, uno que emerge en situaciones emocional y motivacionalmente significativas, vinculadas al aspecto cálido de las FE -dependientes de la corteza orbitofrontal- y otro, el aspecto frío de las FE -dependiente de la corteza prefrontal dorsolateral-, que se activa ante problemas más abstractos, descontextualizados y de baja demanda emocional. En situaciones normales de desarrollo y a pesar de asociarse con distintas partes del cerebro, las FE frías y cálidas trabajan juntas (Zelazo et al., 2005).

La AR y las FE cumplen un papel central en los aprendizajes académicos durante la etapa escolar, y de hecho, el desarrollo de las FE durante la etapa preescolar es un buen predictor de la adaptación al entorno escolar (Whitebread \& Basilio, 2012) como de los futuros logros académicos (Duncan et al., 2007).

\section{Relación entre juego dramático y el desarrollo de la autorregulación}

El papel del juego dramático en el desarrollo cognitivo, ha sido objeto de polémica. En un esfuerzo de síntesis Smith (2010) plantea tres posibles formas de analizar la contribución del juego al desarrollo:

Podría ser un epifenómeno del desarrollo cognitivo, que no tiene importancia real, sino que existen otros aspectos asociados a este tipo de juego que son los que influyen en el desarrollo; podría ser un medio entre otros muchos de favorecer el desarrollo cognitivo, pero no sería el único (equifinalidad); podría ser causalmente necesario para el desarrollo, de modo que la ausencia de juego tuviera consecuencias negativas en algunos aspectos del desarrollo (papel causal).

Tomando como referencia la propuesta de Smith (2010) Lillard et al. (2013) realizan una revisión de la literatura sobre las relaciones entre juego y desarrollo cognitivo, en la que se identifican once dominios -creatividad, inteligencia, solución de problemas, razonamiento, conservación, teoría de la mente, habilidades sociales, lenguaje, habilidades narrativas, funciones ejecutivas y regulación emocional-, en los que la posible influencia del juego ha sido estudiada. $\mathrm{Su}$ revisión señala que, de los once dominios relevados, únicamente en cuatro no es posible descartar la hipótesis del papel causal, a saber: el lenguaje, habilidades narrativas, razonamiento deductivo y regulación emocional. Al mismo tiempo señalan que la evidencia disponible es más consistente con la equifinalidad -donde el juego de simulación es una de las muchas alternativas para lograr el desarrollo-, y con la hipótesis del juego como epifenómeno del desarrollo. Al mismo tiempo afirman que en educación inicial los programas basados en la adquisición de habilidades mediante el juego son más positivos y favorecen el desarrollo más que metodologías tradicionales.

Otra revisión realizada por Nicolopoulou \& Ilgaz (2013) plantea que las investigaciones sobre juego y desarrollo aportan sólidas evidencias del beneficio que se obtiene a partir del juego de simulación, en particular sobre el desarrollo de las habilidades narrativas, y consideran que la participación de los niños en los juegos de simulación brinda muchos elementos que generan beneficios en el desarrollo y que no pueden ser explicados como epifenómenos 
de la intervención del adulto. Plantean también que la investigación de Lillard et al. (2013) es incompleta ya que no recoge varios estudios cruciales para el testeo de la hipótesis causal.

Bodrova et al. (2013) analizando críticamente la revisión de Lillard et al. (2013), señalan que la contribución del juego al desarrollo no debe evaluarse por la mera existencia de juego, sino más bien, por el grado de madurez que alcanza la actividad lúdica. La asociación entre juego y autorregulación sería positiva en la medida en que el juego adopte las características de juego maduro.

La disparidad de resultados de investigaciones con esta temática amerita continuar con los esfuerzos de síntesis para comprender las relaciones entre juego dramático y autorregulación. Por esta razón el objetivo de la presente revisión sistemática es relevar la investigación sobre las relaciones entre juego dramático y desarrollo de la autorregulación/ funciones ejecutivas en la edad preescolar con especial interés en identificar la direccionalidad de esa relación.

\section{Método}

Para la presente revisión se recurrió al Portal Timbó (Trama Interinstitucional Multidisciplinaria de Bibliografía Online), de la Agencia Nacional de Investigación e Innovación (ANII), Uruguay. Se trata de un portal institucional que permite la realización de búsquedas en simultáneo en múltiples colecciones y bases de datos multidisciplinarias como Ebsco-host, Jstor, Sage publishing, Science direct, Scopus y Springer link.

\section{Estrategia de búsqueda $y$ criterio de selección}

Se realizaron dos búsquedas el 23 de marzo de 2020, la primera de ellas incluyó artículos escritos en español e inglés, las palabras utilizadas fueron play, selfregulation y preschool, -se hizo la búsqueda en inglés dado que no se encontraron resultados relevantes tras búsquedas en español-. No hubo limitación temporal y se dispuso que las palabras estuvieran en el resumen, esta búsqueda arrojó un total de 124 artículos.

Se consideraron los siguientes criterios de inclusión: primero, los estudios debían examinar la posible relación entre el juego dramático y el desarrollo de la AR o las FE, por ende debían ser investigaciones empíricas en niños de edad preescolar; segundo, las muestras debían incluir niños de desarrollo típico de entre 3 y 6 años; tercero, los niños participantes de dichas investigaciones debían estar escolarizados, dado que el interés radica en aportar conocimiento a la hora de pensar la forma de trabajar con grupos de estas edades.

Tras considerar únicamente las publicaciones académicas y eliminar las repeticiones exactas, el resultado fueron 52 artículos. Con la lectura del título y resumen se eliminaron los trabajos no relacionados y los que no fueran investigaciones empíricas, lo que resultó en 4 artículos a revisar: Elias \& Berk, 2002; Nicolopoulou, Schnabel Cortina, Ilgaz, Brockmeyer Cates \& de Sá, 2015; Slot et al., 2017; Vieillevoye \& NaderGrosbois, 2008.

Se realizó una segunda búsqueda cambiando la expresión self-regulation por executive function, con las mismas 
condiciones, el resultado fueron 141 artículos de los cuales solo quedaron 68 luego de imponer las limitaciones y de éstos únicamente 2 resultaron pertinentes para esta investigación: Carlson et al., 2014 y Thibodeau, Gilpin, Brown \& Meyer, 2016. Finalmente, tras revisar las referencias de estas investigaciones, se sumó el artículo Kelly \& Hammond, 2011 que se menciona en la investigación realizada por Carlson, et al. (2014).

\section{Resultados}

Siete artículos fueron incluidos en esta revisión. La tabla 1 reseña cada uno, incluyendo la muestra que se utilizó, el método, los instrumentos, las variables de interés y los resultados obtenidos.
Las muestras son homogéneas en varios aspectos: en la franja etaria seleccionada, todos los estudios incluyen niños de edad preescolar; en cuanto al nivel socio económico de las familias, siendo éstas de nivel medio en seis estudios, solo un estudio -el 7- se realiza sobre niños pertenecientes a familias de bajos recursos; $\mathrm{y}$ en cuanto al estatus de desarrollo de los participantes, con seis estudios realizados sobre niños con desarrollo típico y uno -el 2que incluye participantes de desarrollo típico y atípico -discapacidad intelectual-.

Los estudios 1, 3, 4 y 7 fueron realizados en instituciones educativas, pudiendo en estos casos observarse el comportamiento de los niños en situaciones naturales de juego para el contexto educativo, mientras que los estudios 2,5 y 6

Número de registros identificados en la primera búsqueda (play, self-regulation, preschool) /

Número de registros identificados en la segunda búsqueda (play, executive function, preschool)

$\downarrow$

Número de registros luego de aplicar filtros de búsqueda

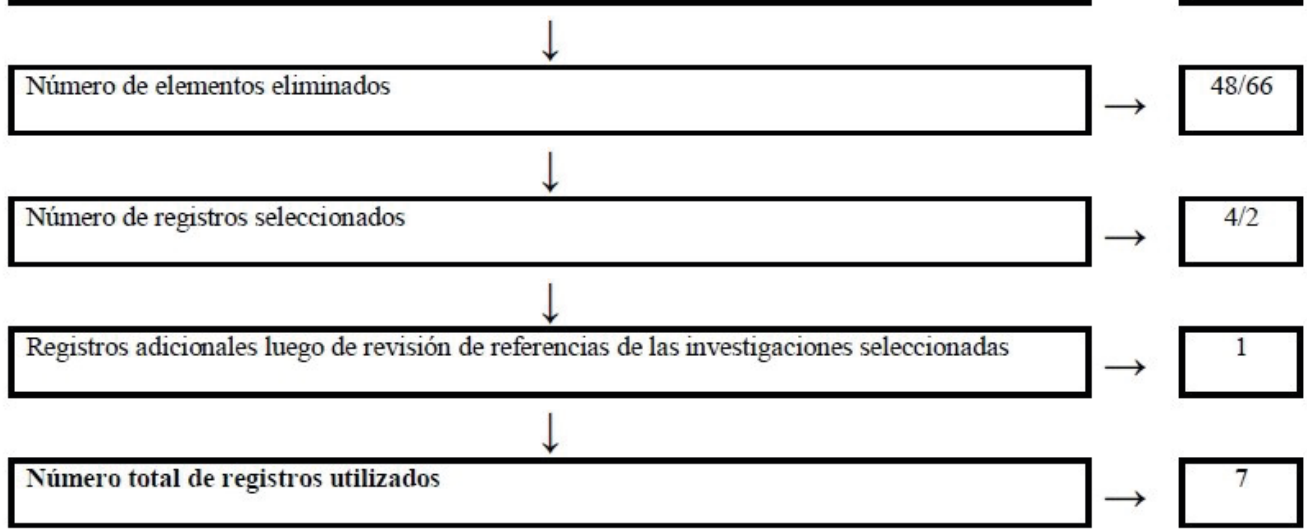

Figura 1. Diagrama de flujo de la revisión (se separan con una barra (/) los resultados de la primera y segunda búsqueda) 


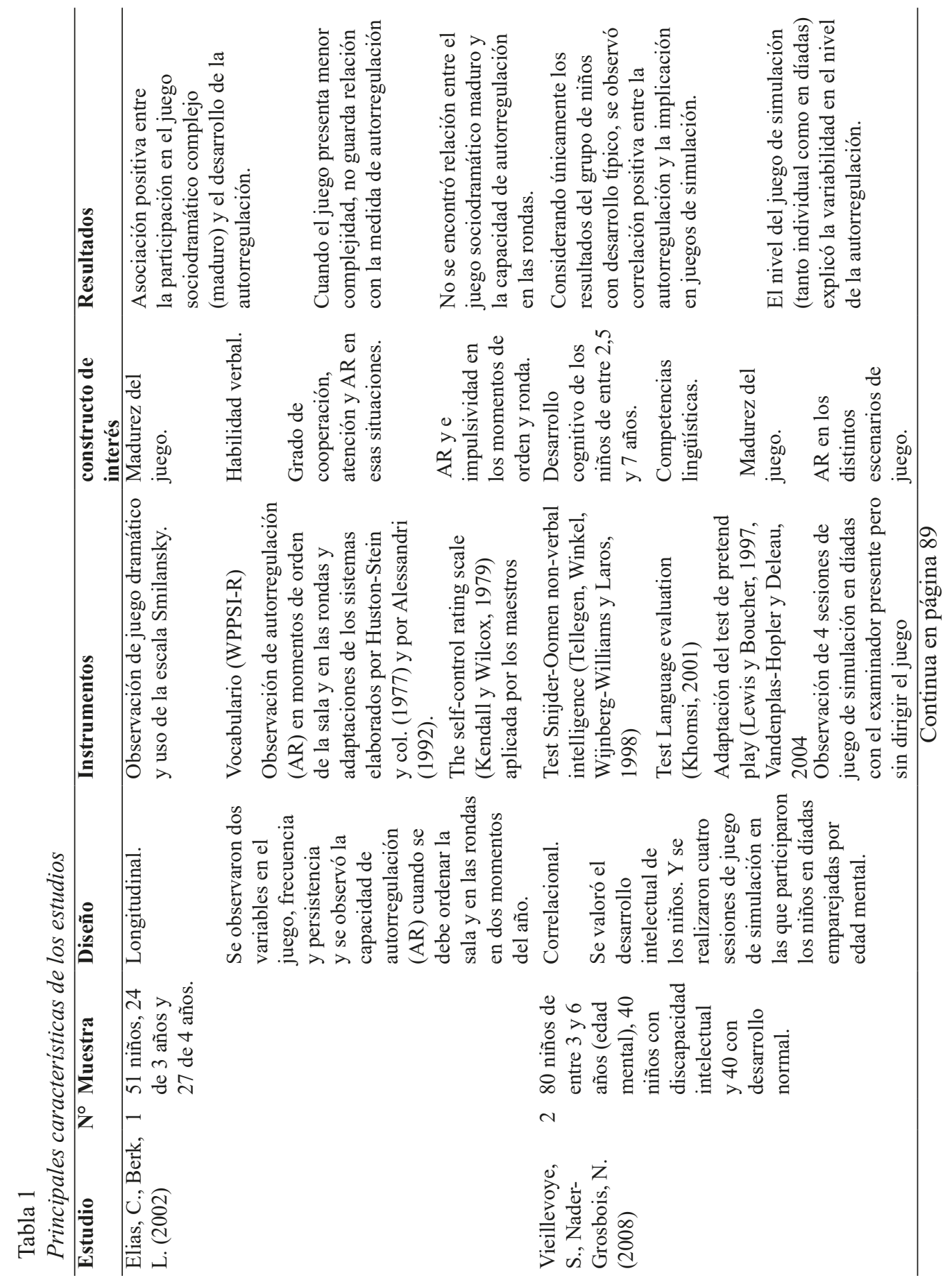




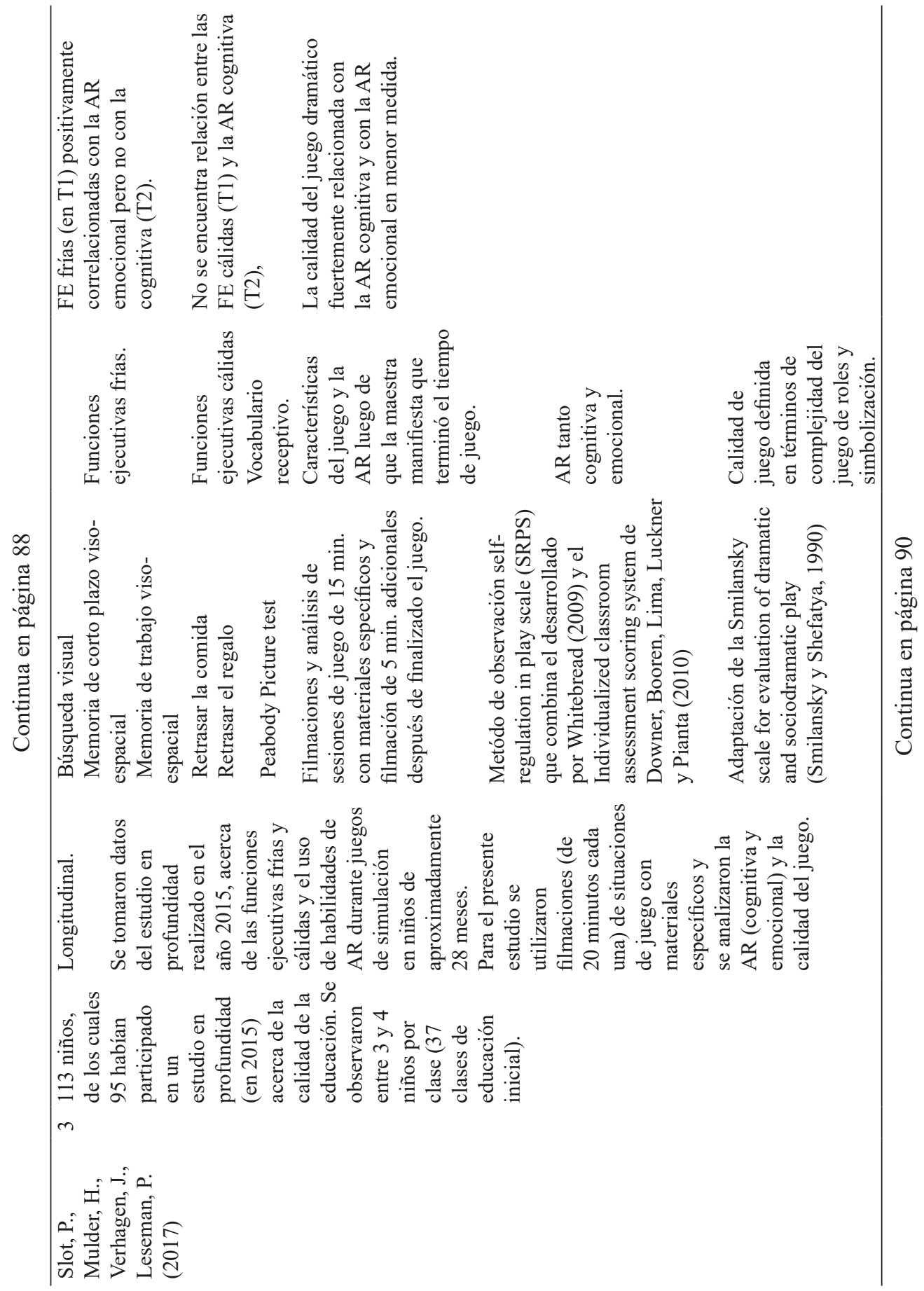

Revista de Psicología. Año 2020. Vol. 16, No 32, pp. 79-103 


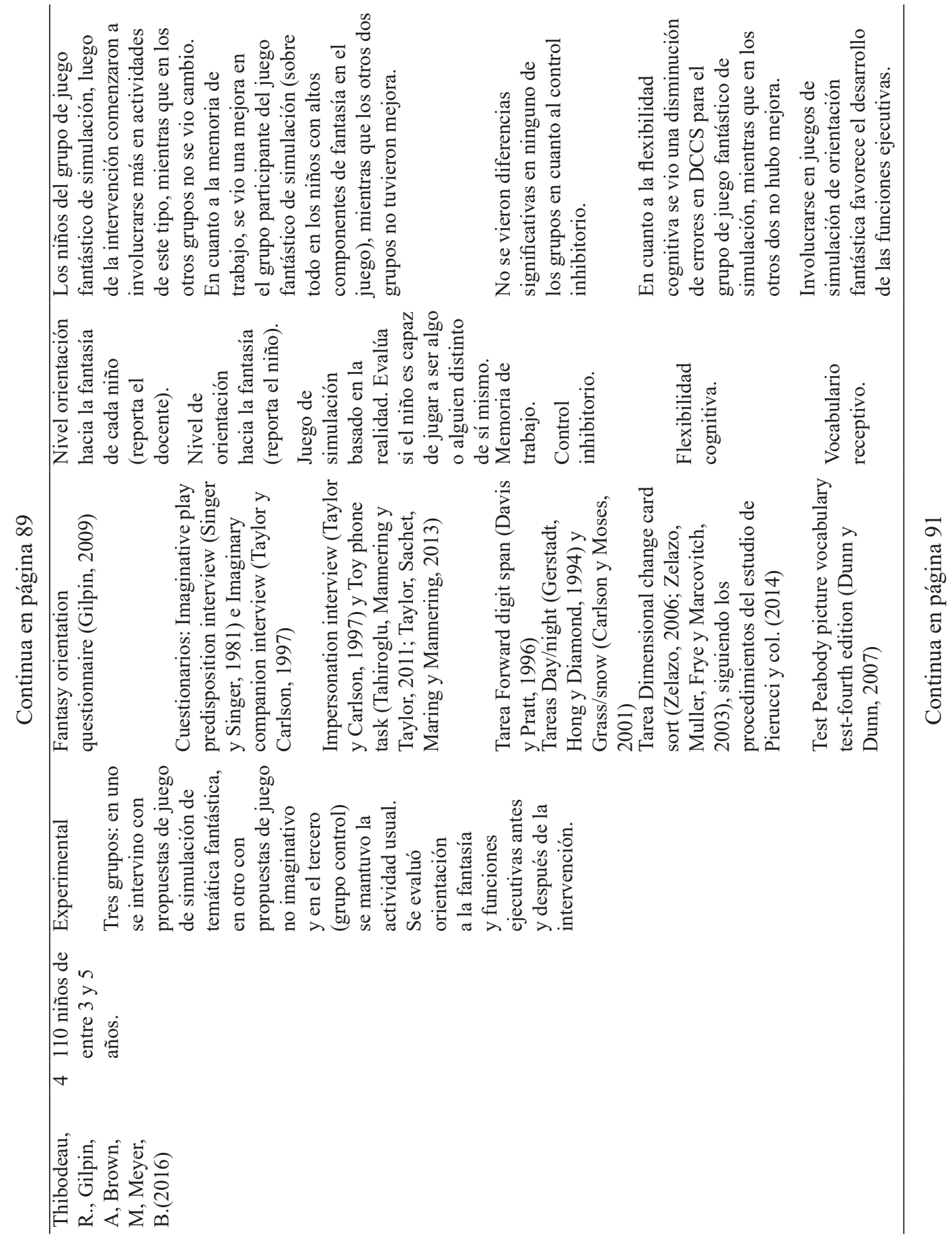


Las relaciones entre juego dramático y autorregulación: Una revisión sistemática

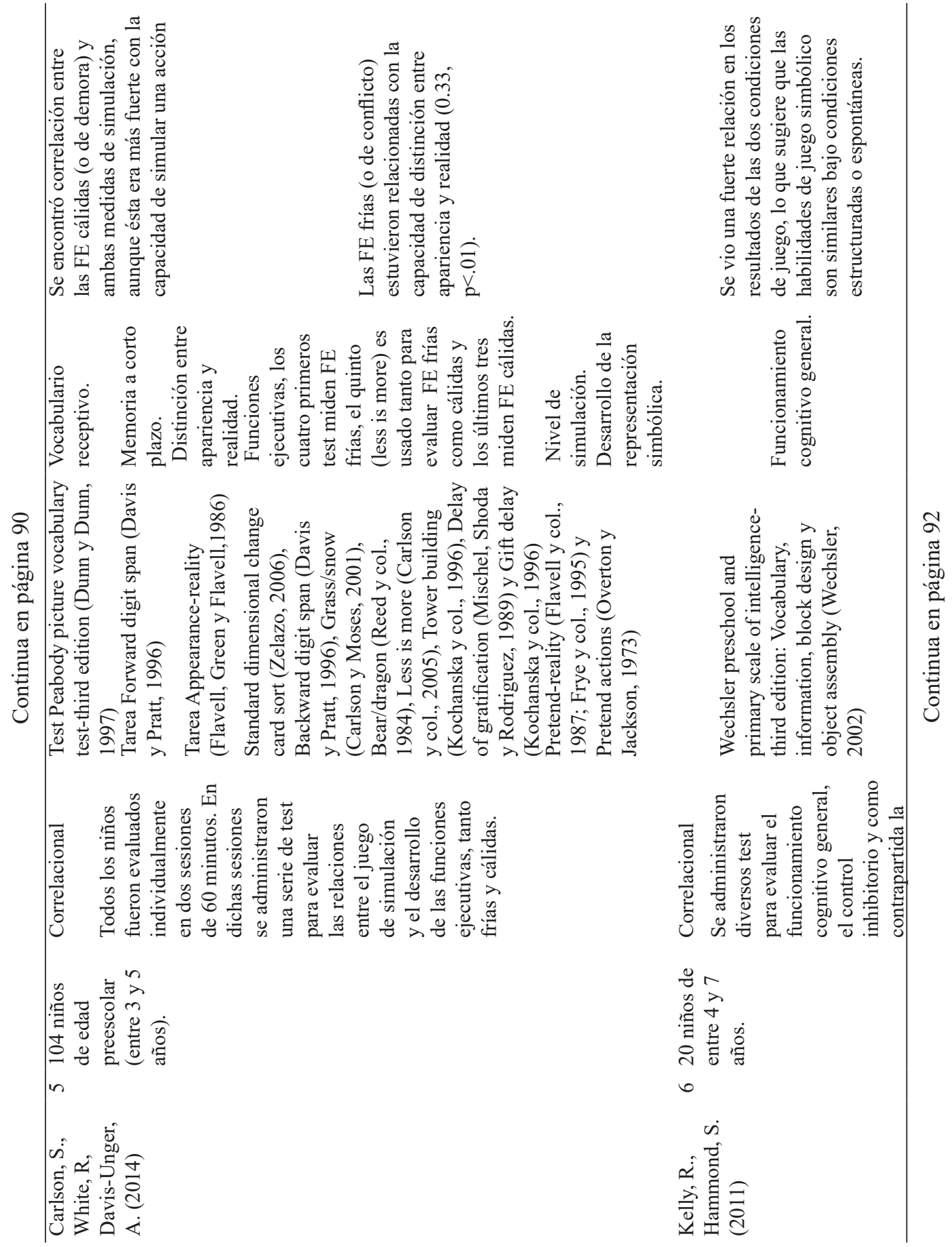

Revista de Psicología. Año 2020. Vol. 16, № 32, pp. 79-103 


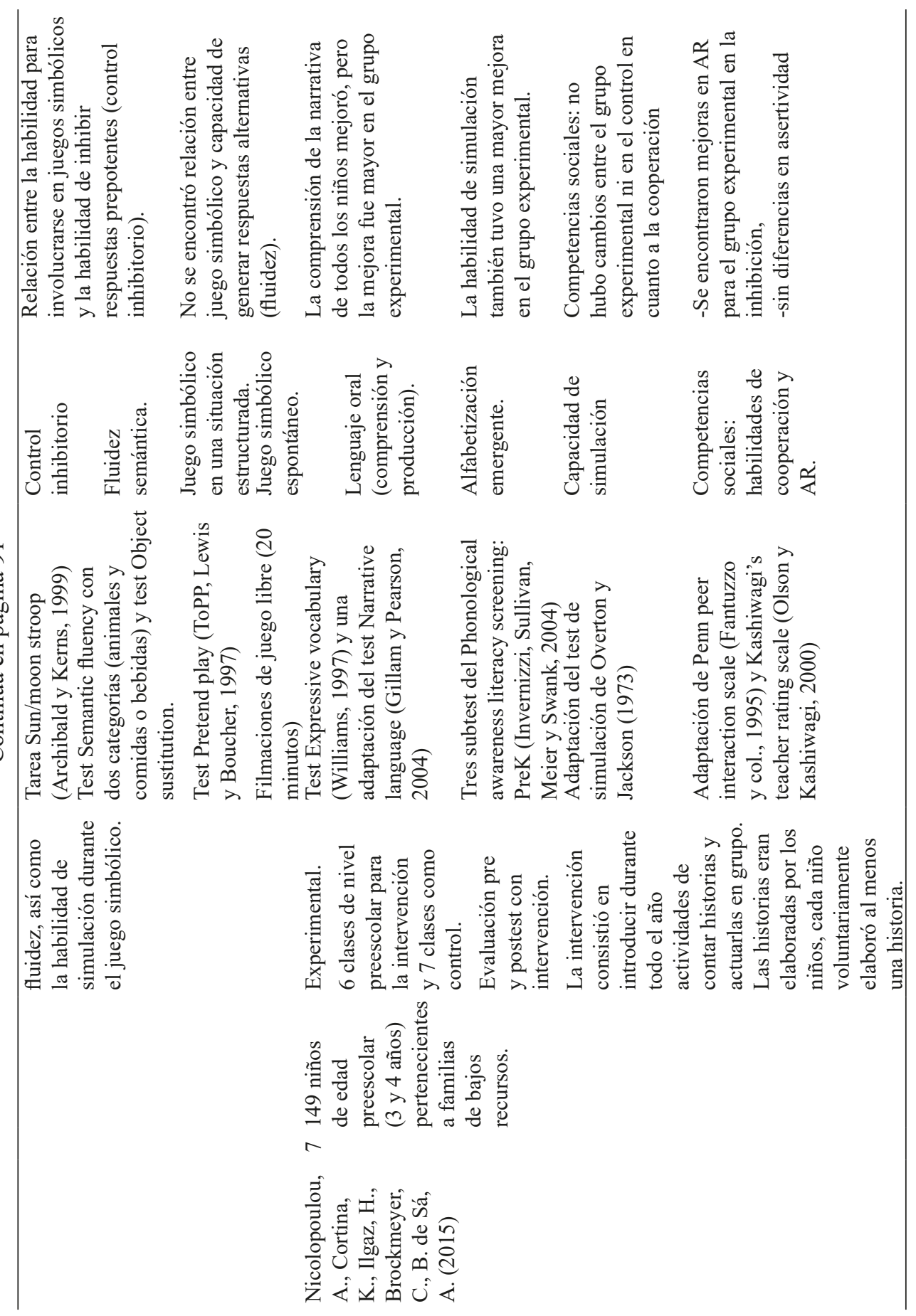


se desarrollaron en contexto de laboratorio.

La mayor parte de los estudios son de observación y utilizan diseños transversales $-2,5$ y 6-, el estudio 1 utiliza un diseño longitudinal, el 3 uno correlacional y los estudios 4 y 7 son de intervención, con un diseño es de pre test - post test.

Todos los estudios consideran el funcionamiento cognitivo de los sujetos al momento del comienzo del estudio. Lo evalúan a través de test de habilidad verbal, el WPPSI -1 y 6-, el Peabody Picture Vocabulary Test -3, 4 y 5-, el Test Language Evaluation de Khomsi -2- y el Test Expressive Vocabulary de Williams y el Narrative Language de Guillam y Pearson -7-. El funcionamiento cognitivo es una variable que debe controlarse para poder comparar resultados, por este motivo el estudio 2 incluyó participantes en situación de discapacidad intelectual emparejados con niños de desarrollo típico por su edad mental.

Se presenta a continuación una síntesis de resultados, respondiendo en forma independiente cada una de las preguntas de investigación, y analizando el aporte de cada uno de los estudios.

\section{Sobre la evaluación de los constructos autorregulación y funciones ejecutivas}

Los estudios revisados presentan una cobertura dispar en cuanto a la evaluación de la AR y las FE. Los 1, 2, y 7 desde la perspectiva de sus autores, se enfocan en la $\mathrm{AR}$, evaluando habilidades de cooperación, atención e impulsividad. Los estudios 4, 5 y 6 se enfocan en el funcionamiento ejecutivo y evalúan memoria de trabajo, control inhibitorio y flexibilidad cognitiva -estudio 4-, memoria a corto plazo y habilidades de autocontrol -estudio 5- y control inhibitorio -estudio 6-. Por su parte, el estudio 3 se enfoca en ambos constructos y evalúa $\mathrm{FE}$ frías y cálidas, y AR cognitiva y emocional. Cabe señalar que ninguno de los estudios realiza una evaluación integral del funcionamiento ejecutivo o la autorregulación, sino que toman solamente algún aspecto de estos.

\section{Definiciones de juego dramático en las investigaciones analizadas}

El estudio 1 se centra en los aspectos del juego dramático que los autores consideran que podrían favorecer el desarrollo de la autorregulación: tiempo que el niño representa el rol que le tocó o que eligió jugar, uso de objetos -que daría cuenta del carácter más o menos imaginativo del juego-, por último analiza la interacción entre los participantes del juego, que debe estar mediada por comunicación verbal cuya meta sería prolongar el episodio de juego. De este mismo modo se evalúa el juego en el estudio 7 , viendo en qué medida la actuación de los niños se ajusta a las reglas impuestas en el juego y la mediación del lenguaje en el juego. El estudio 2 se enfoca en los roles, las acciones, el modo de utilizar los objetos y la participación social, además del grado de involucramiento tanto en niños que juegan solos como en aquellos que se observa jugando en duplas. En los estudios 3, 4, 5 y 6 la mirada del investigador está puesta en la calidad o complejidad del juego. El juego se va complejizando a medida que se va volviendo más flexible, creativo y en cierto modo más abstracto, menos dependiente de lo que se percibe en el entorno. En este tipo de juegos se observa una base común con ciertas reglas, sobre la que se coordinan 
los distintos roles y los objetivos del juego, se negocian planes de acción, se debe monitorear el juego y adaptar las acciones en base a lo que van haciendo sus compañeros.

\section{Instrumentos utilizados para evaluar el juego}

Dado que se pretende estudiar la relación entre el juego dramático y el desarrollo de la AR, es importante observar de qué forma mide cada estudio este constructo. Los instrumentos que se utilizan en los estudios analizados para evaluar el juego de los participantes son diversos, los estudios 1,3 y 6 utilizan la observación del juego dramático y tanto el estudio 1 como el 3 utilizan la escala Smilansky para su evaluación (Smilansky \& Shefatya, 1990). Los estudios 2 y 6 valoran la madurez del juego con una adaptación del test pretend play (Lewis \& Boucher, 1997; VandenplasHolper \& Deleau, 2004). El estudio 4 se interesa por el componente de fantasía en el juego y lo valora mediante cuestionarios propuestos tanto a los maestros como a los niños (Gilpin, 2009; Singer \& Singer, 1981; Taylor \& Carlson, 1997). El estudio 5 se interesa en la capacidad de simulación y la valora a partir de los test Pretend-reality (Flavell, Flavell \& Green, 1987; Frye, Zelazo \& Palfai, 1995) y Pretend actions (Overton \& Jackson, 1973), el 7 también evalúa la capacidad de simulación pero para esto utiliza únicamente la tarea de pantomima de Overton y Jackson (1973), otro aspecto que evalúa este estudio son las competencias sociales en el juego y para esto utiliza el Penn Peer Interaction Scale children (Fantuzzo et al., 1995). A pesar de que todos estudian el juego en relación con el desarrollo de la AR o FE, todos lo estudian empleando metodología diferente y no todos consideran un aspecto tan relevante como es la calidad del juego.

\section{Influencia del juego dramático en el desarrollo de la autorregulación}

Todos los estudios revisados reportan que involucrarse en juegos de simulación con determinadas características está directamente relacionado con el desarrollo de la AR o con algún aspecto de las FE. Sin embargo, no se pudo llegar a resultados concluyentes acerca de la dirección causal de dicha relación -si es el juego maduro el que favorece el desarrollo de la AR o es el alcanzar determinado nivel en cuanto a la AR o en las FE las que favorecen la adquisición de determinados tipos de juego-. Los estudios 1, 2, 3 y 7 exploran el rol del juego de simulación en el desarrollo de la AR, los estudios 4 y 5 exploran el rol del juego en el desarrollo de algún aspecto de las FE, mientras que el estudio 6 explora el rol de las FE -particularmente control inhibitorioen el desarrollo del juego simbólico. En los estudios 3, 5 y 6 se explicita que, si bien puede establecerse una clara relación entre estos constructos, una de las limitaciones de estos estudios radica justamente en que la direccionalidad de esta no puede asegurarse. Únicamente en el estudio 4 se examina la direccionalidad de esta relación y concluye que el juego con orientación a la fantasía es una de las actividades que favorece el desarrollo de las FE.

Uno de los estudios -2- plantea que el juego de simulación promueve el desarrollo de la creatividad, y ésta favorece la capacidad de planificar, de resolver problemas y la AR 
en actividades subsiguientes.

Otro de los estudios -3- distingue entre AR emocional y cognitiva, observando que el juego dramático, luego de que alcanza determinado nivel, está relacionado con ambas, influenciándolas positivamente.

Por último, el estudio 5, menciona que el juego dramático, al contribuir al desarrollo del pensamiento simbólico, ayuda al niño a distanciarse de estímulos irrelevantes para la situación planteada, con lo que se fortalece la AR. Los niños a través de este tipo de juego aprenden a pensar de manera más flexible y reflexiva, habilidad necesaria para la regulación cognitiva y emocional.

\section{Las características del juego ligadas al desarrollo de la autorregulación}

Al hablar de juego en relación con el desarrollo de las FE o de la AR, parece haber consenso en que no todo tipo de juego lo promueve. Los estudios que evalúan la calidad o el grado de madurez del juego son el 1, 2 y 3, llegando a concluir que solamente con un juego dramático complejo o maduro se logra favorecer el desarrollo.

En el estudio 3, se considera que el juego es de alta calidad cuando el niño puede representar un personaje elaborado durante todo el episodio de juego, cuando es capaz de utilizar sustitución de objetos, de describir verbalmente acciones o situaciones, cuando hay interacciones, ya sea a través de palabras o acciones, dentro de la situación de juego -con pares o adultos- y cuando se observa metacomunicación, que se refiere a la capacidad de entrar y salir del juego, por ejemplo cuando se necesita asignar roles o discutir acerca del curso del juego. También se menciona en este estudio que el juego de simulación requiere que los niños coordinen sus metas, negocien planes, hagan un constante monitoreo del progreso del juego y lo que los demás niños hacen para adaptar sus acciones a la situación que se esté dando.

En el estudio 4 se menciona la calidad del juego para referirse a qué tan involucrado está el niño en el juego. Y considera muy importante el componente de fantasía de los juegos de simulación -siendo éste uno de los aspectos del juego que da cuenta de su maduración- percibiéndose una relación directa con el desarrollo de algunas FE -memoria de trabajo y flexibilidad cognitiva-. El estudio 5 también hace referencia a los elementos de fantasía que incluyen algunos niños en sus juegos. También se plantea en éstas investigaciones que más allá del estímulo que reciban los niños para lograr este tipo de juego, también influyen sus características personales, ya que algunos niños desarrollan más fácilmente escenarios fantásticos y otros juegan en escenarios más realistas.

En los estudios 1, 3 y 6 si bien no se habla de fantasía, se hace referencia al componente de imaginación del juego dramático, gracias al cual los niños perciben la situación actual, pero simulan que existe una realidad diferente, pudiendo discriminar ambas situaciones -real e imaginaria-, y logran modificar la identidad y el significado de los objetos o situaciones.

Los estudios 2 y 7 no mencionan el papel de la fantasía en el juego.

\section{Sobre la direccionalidad de la relación entre juego y autorregulación}

Para el análisis de la direccionalidad de las relaciones entre juego y AR se analizó 
el tipo de evidencia aportada.

Los estudios 1, 2, 3, 5 y 6 si bien muestran una asociación consistente entre juego y AR, no permiten testear su direccionalidad, ya que aportan evidencia correlacional.

En el estudio 1 se plantea que los resultados de la investigación son consistentes con la teoría de Vygotsky en cuanto a la importancia del juego sociodramático en el desarrollo de la autorregulación, y agregan que incluso los niños impulsivos -que tienen menos desarrollada su capacidad de autorregulación- se benefician enormemente de estas situaciones de juego.

El estudio 2 plantea que el uso de los juegos dramáticos como estrategia mejora la forma en que los niños resuelven problemas, además de que promueve la planificación y la AR. En el juego los niños están constantemente focalizando su atención, seleccionando entre posibles formas de actuar, inhibiendo conductas que no son adecuadas, planificando, respondiendo a demandas sociales, resolviendo problemas, entre otras acciones. Por esto se puede ver al juego como una actividad que brinda al niño variadas oportunidades para practicar $y$ adquirir la AR.

El estudio 3 encuentra que la calidad del juego está fuertemente relacionada a la AR cognitiva y también está asociada, aunque en menor medida, a la AR emocional, enfatizando que no todo tipo de juego promueve el desarrollo, la calidad de este es un aspecto destacado. En este sentido Bodrova et al., (2013) también elaboran una respuesta al artículo de Lillard et al. (2013), donde plantean que para poder estudiar la relación que existe entre el juego de simulación y el desarrollo es fundamental poder diferenciar entre juego maduro e inmaduro dado que no todo tipo de juego promueve desarrollo. Consideran estas autoras que es en este punto donde fallan algunos estudios y aportan herramientas para poder juzgar la calidad de juego de simulación del niño.

En los estudios 5 y 6 también se encuentra relación entre el juego simbólico, tanto bajo condiciones estructuradas como espontáneas -que implica capacidad de simulación y habilidad para manejar representaciones duales- y el desarrollo de las FE, de acuerdo con el estudio 6 esta relación se da con el control inhibitorio particularmente.

Dos de los estudios revisados, el 4 y el 7, van más allá del estudio de la asociación entre juego y AR, apuntando al tema de la direccionalidad. Los resultados del estudio 4 son compatibles con la hipótesis de equifinalidad. El grupo de niños involucrado en juego dramático de tipo fantástico tuvo mayores mejoras en las FE que el grupo de niños en los que se intervino con propuestas de juego no imaginativo o el grupo control donde no se intervino. De este modo, concluye que involucrar a los niños en juegos de este tipo es una de las formas en las que podemos influir directamente en el desarrollo de las FE, y si bien hay otros modos de influir en este desarrollo, el juego dramático de tipo fantástico es altamente favorable. En este sentido, la hipótesis causal y la de equifinalidad no resultan excluyentes, pues como plantean Nicolopoulou \& Ilgaz (2013), la equifinalidad también es un tipo de relación causal en tanto que el juego es uno de los factores que promueve el desarrollo de las FE aunque no sea el único. En la misma dirección, Kroll (2016) plantea que hay varias 
categorías de acciones que influyen en el desarrollo de la AR. El juego es una de ellas, y tiene especial relevancia, pero también se destacan las rutinas, el currículum y algunos aspectos del medioambiente. En cuanto al comportamiento de los niños en clase, este autor hace una distinción interesante entre aquellos niños que están desarrollando la $\mathrm{AR}$ y aquellos que simplemente son dóciles. En ambos casos el niño "se porta bien", pero es trabajo del docente notar si la regulación se da en un plano interno -que sería la AR- o en un plano externo, sólo cuando alguien le pide al niño que se comporte de determinada manera -con docilidad-. Es haciendo esta diferenciación que el docente puede ayudar al niño a desarrollar la AR y el juego es la herramienta privilegiada. Díaz, Neal \& Amaya-Williams, (2003) van más allá y plantean que es importante también diferenciar entre autocontrol y AR, siendo que cuando el niño pone en práctica el autocontrol está cumpliendo una orden o un pedido en ausencia del adulto -responde a una orden de un tutor internalizado-, por lo que en este caso también la regulación se estaría dando desde un plano externo aunque el adulto no esté presente, sin embargo cuando el niño logra la AR, su conducta sigue un plan de acción formulado por sí mismo. El autocontrol sería entonces un paso previo al logro de la AR. Proponen los autores que la regulación de la conducta es en una primera instancia un fenómeno interpersonal y destacan la importancia de la interacción con los adultos en el desarrollo de la AR.

En el estudio 7, cuya modalidad es de intervención, se encontró que la actividad de crear historias y actuarlas mejora la habilidad de simulación y favorece el desarrollo de la $\mathrm{AR}$, específicamente en lo que refiere a la inhibición. Para este estudio se tomaron 13 clases de nivel preescolar, se intervino en 6 de ellas y se tomaron las 7 restantes como grupo de control. La intervención consistió en introducir actividades durante todo el año en las cuales los niños debían crear historias, contarlas y actuarlas en subgrupos -cada niño elaboró voluntariamente al menos una historia-. Se evaluó al grupo pre y postest para llegar al resultado de que tanto la comprensión de la narrativa, como la habilidad de simulación y la inhibición -en lo que respecta a la AR- tuvieron mayores mejoras en los grupos en los que se intervino que en los de control. Con lo que el resultado de este estudio coincide con el del estudio 4 en cuanto a la hipótesis de equifinalidad.

Nicolopoulou et al. (2015) en el estudio 7 también plantean que el juego requiere y a la vez promueve la AR.

\section{Discusión}

El objetivo de este trabajo fue revisar la evidencia disponible sobre las relaciones entre juego dramático y autorregulación y especialmente sobre la direccionalidad de esta relación en la edad preescolar, cuando el juego dramático es la actividad rectora del desarrollo. Para ello tomamos como punto de partida la categorización de Smith (2010) sobre las funcionalidades evolutivas del juego y la revisión de Lillard, et. al (2013). Seis de los siete estudios que integran nuestra revisión no fueron reportados en el trabajo de Lillard et. al (2013).

Se ha afirmado que el juego ayuda a los niños a subordinar sus deseos a las reglas sociales, a cooperar voluntariamente con otros y a entablar un comportamiento social apropiado en cada situación. El juego 
dramático o juego de roles contribuye en la adquisición de competencias sociales, incluyendo la AR y la capacidad de manejar las emociones (Bodrova et al., 2013; Elías \& Berk, 2002; Elkonin, 1980; Hirsh-Pasek, Golinkoff, Berk \& Singer, 2009; Karpov, 2005; Paley,1990; Vygotski, 1996; 2000). A partir de los resultados de los estudios revisados podemos concluir que el involucrarse en situaciones de juego dramático se vincula con el desarrollo de la $\mathrm{AR}$ o las FE, necesarias para una inserción exitosa en el ciclo de educación primaria y para la vida en general. A pesar de ello no estamos en condiciones de determinar si es el juego lo que conduce a formas más maduras de $\mathrm{AR}$, o si, por el contrario, una AR más desarrollada lleva a formas más maduras de juego. También es posible pensar que las relaciones sean de influencia mutua, más que de influencia unidireccional de uno a otro. En este sentido Foley (2017) plantea que "La etapa del juego simbólico es el punto más alto durante el cual, el juego y la regulación se vuelven mutuamente interdependientes, una promueve a la otra." (p. 245) En su revisión de la investigación muestra cómo favoreciendo el desarrollo de uno de estos procesos, influenciamos el desarrollo del otro, con lo que esta relación podría ir en ambas direcciones.

Un aspecto poco atendido en la literatura revisada es el de la calidad del juego, en términos del grado en el que los niños se comprometen en situaciones de juego maduro. Smirnova y Gudareva (2015) plantean que, con las actuales situaciones imaginarias poco desarrolladas y los roles y reglas pobremente especificados, este juego "inmaduro" no concuerda con el tipo de juego que Vygotsky y sus seguidores identificaron como una fuente de desarrollo. En el estudio que llevaron a cabo observaron que muy pocos niños alcanzan formas maduras de juego, y que esto se está dando principalmente en niños de seis años o más, ya finalizando la edad preescolar. También observaron que al juego en educación preescolar se le está dando un valor casi exclusivamente didáctico, se utiliza el juego como herramienta para enseñar habilidades y conceptos, siendo que ésta no sería la función principal del juego, sino que lo realmente significativo es lo que el juego puede aportar al desarrollo y a la formación de la personalidad del niño. "En su juego, los niños aprenden a controlarse y evaluarse a sí mismos, a entender qué están haciendo y -probablemente lo más importante- a querer actuar correctamente... hace a su comportamiento consciente y voluntario." (Smirnova \& Gudareva, 2015, p.18). En este mismo sentido, Bredekamp (2005) plantea que se necesita de la intervención del adulto o de pares más capaces para lograr un tipo de juego maduro que involucre situaciones imaginarias, roles, reglas y que esté mediado por interacciones lingüísticas prolongadas.

\section{Limitaciones}

Las principales limitaciones de este estudio fueron: el número de estudios empíricos encontrados y la dificultad en comparar estos estudios debido a la diversidad en la forma de abordar los constructos de interés. A pesar de que en todos los estudios se trabaja con el juego como herramienta para promover desarrollo, no todos se interesan en los mismos aspectos del juego, por ejemplo, no todos se interesan por la madurez del juego. Una limitación adicional refiere al tipo de 
diseño bajo el que se estudian las relaciones entre juego y $\mathrm{AR}$, que en su mayor parte fueron correlacionales, lo que no permite responder preguntas sobre la influencia causal entre ambos.

\section{Direcciones futuras}

A partir de los estudios analizados se obtuvo evidencia, fundamentalmente correlacional sobre las relaciones entre juego y AR/FE. Por ello se plantea la necesidad de estudios que avancen en el análisis de la direccionalidad de la relación entre ambos constructos; o, lo que es equivalente, si conseguir avances en el desarrollo de la AR de forma no lúdica impacta en la calidad de juego. Si al influir en el desarrollo de la AR/FE, también influimos en la forma en que el niño juega, la relación sería causal en ambos sentidos y si el juego no sufre modificaciones, quedaría demostrado que la relación es causal sólo en un sentido -que el juego influye en el desarrollo de la AR/FE pero no viceversa-. La investigación futura debería atender este aspecto para poder tener un mayor entendimiento de lo que implica la relación entre el juego dramático maduro y el desarrollo de la AR.
Dilucidar la direccionalidad de la relación entre juego y AR, permitirá avanzar en formas adecuadas de intervención en situaciones educativas. Si se logra mostrar que el juego dramático promueve el desarrollo de la $\mathrm{AR} / \mathrm{FE}$, sería razonable tender al diseño de intervenciones que promuevan el desarrollo de la AR a través del juego. Estas intervenciones podrían enfocarse en la promoción de patrones de juego maduro.

Si bien el foco de nuestra revisión no eran las intervenciones adultas, algunos de los estudios revisados se enfocaron en situaciones de juego en el aula -1, 3, 4 y 7-, y describen al adulto como un actor presente en el juego, e interviniendo cuando se plantea un conflicto que los niños no pueden solucionar por sí mismos o para aportar al desarrollo del juego. Sería importante, en vista de un futuro análisis, estudiar en profundidad el papel de los adultos referentes y de los pares tanto en la evolución del juego como en el desarrollo de la AR/FE, ya que la literatura previa ha mostrado que juegan un rol relevante en su desarrollo (Bernier, et al., 2011; Bibok, et. al, 2009; Hughes, 2013; Moriguchi, 2014).

\section{Referencias}

Bernier, A., Carlson, S. M., Deschênes, M., \& Matte-Gagné, C. (2011). Social factors in the development of early executive functioning: a closer look at the caregiving environment. Developmental Science, 15(1), 12-24. doi:10.1111/j.14677687.2011.01093.x
Bibok, M. B., Carpendale, J. I. M., \& Müller, U. (2009). Parental scaffolding and the development of executive function. New Directions for Child and Adolescent Development, 2009(123), 17-34. doi:10.1002/ cd.233

Blair, C. \& Raver, C. (2015). School 
readiness and self-regulation: A developmental psychobiological approach. Annu. Rev. Psychol., 66, 711 - 731. Doi: 10.1146/annurevpsych-010814-015221

Bodrova, E. (2008). Make-believe play versus academic skills: a Vygotskian approach to today's dilemma of early childhood education, European Early Childhood Education Research Journal, 16(3), 357-369, Doi: $10.1080 / 13502930802291777$

Bodrova, E. \& Leong, D. (2004). Herramientas de la mente. El aprendizaje en la infancia desde la perspectiva de Vygotsky. México: Pearson educación de México.

Bodrova, E., Leong, D., \& Akhutina, T. V. (2011). When everything new is well-forgotten old: Vygotsky/ Luria insights in the development of executive functions. New Directions for Child and Adolescent Development, (133), 11-28. https:// doi.org/10.1002/cd

Bodrova, E., Germeroth, C. \& Leong, D. (2013). Play and self-regulation: Lessons from Vygotsky. American Journal of play, 6(1), $111-123$.

Bredekamp, S. (2005). Play and school readiness. Educational perspectives, $38(1), 18-26$.

Canet-Juric, L., Introzzi, I., Andrés, M. \& Stelzer, F. (2016). La contribución de las funciones ejecutivas a la autorregulación. Cuadernos de neuropsicología, 10(2), $106-128$.

Carlson, S. (2005) Developmentally Sensitive Measures of Executive Function in Preschool Children,
Developmental Neuropsychology, 28(2), 595-616, Doi: 10.1207/ s15326942dn2802 3

Carlson, S., White, R. \& Davis-Unger, A. (2014). Evidence for a relation between executive function and pretense representation in preschool children. Cognitive development, $1-16$. Doi: 10.1016/j. cogdev.2013.09.001

Diamond, A. (2013). Executive functions. Annu Rev Psychol. 64, 135 168. Doi: 10.1146/annurevpsych-113011-143750.

Diamond, A. (2016). Why improving and assessing executive functions early in life is critical. Executive Function in PreschoolAge Children: Integrating Measurement, Neurodevelopment, and Translational Research., 1143. https://doi.org/10.1037/14797002

Díaz, R., Neal, C \& Amaya-Williams, M. (1998). Orígenes sociales de la autorregulación. En Vygotsky y la educación. (p. 153-185). Buenos Aires. Aique Grupo Editor S.A.

Duncan, G. J., Dowsett, C. J., Claessens, A., Magnuson, K., Huston, A. C., Klebanov, P., Pagani, L. S., Feinstein, L., Engel, M., BrooksGunn, J., Sexton, H., Duckworth, K., Japel, C. (2007). School readiness and later achievement. Developmental Psychology, 43(6), 1428-1446. doi:10.1037/00121649.43 .6 .1428

Elias, C. \& Berk, L. (2002). Self-regulation in young children: is there a role for sociodramatic play? Early 
childhood research quarterly, 17, $216-238$.

Elkonin, D. (1980). Psicología del juego. Madrid: Visor.

Fantuzzo, J. W., Sutton-Smith, B., Coolahan, K. C., Manz, P., Canning, S., \& Debnam, D.(1995). Assessment of play interaction behaviors in young low-income children:Penn Interactive Peer Play Scale. Early Childhood Research Quarterly, 10, 105-120.http://dx.doi. org/10.1016/0885-2006(95)900284

Fernyhough, C. (2010). Vygotsky, Luria, and the Social Brain. In \& G. I. B. Sokol, U. Müller, J. Carpendale, A. Young (Ed.), Self- and SocialRegulation: Exploring the Relations between Social Interaction, Social Cognition, and the Development of Executive Functions (pp. 56-79). Durham University.

Flavell, J. H., Flavell, E. R., \& Green, F. L. (1987). Young children's knowledge about the apparentreal and pretend-real distinctions. Developmental Psychology, 23, 816-822.

Foley, G. (2017). Play as regulation: promoting self- regulation through play. Topics in language disorders, 37(3), $241-258$.

Frye, D., Zelazo, P. D., \& Palfai, T. (1995). Theory of mind and rule-based reasoning. Cognitive Development, $10,483-527$.

Garon, N., Bryson, S. E., \& Smith, I. M. (2008). Executive function in preschoolers: A review using an integrative framework.
Psychological Bulletin, 134(1), 3160. https://doi.org/10.1037/00332909.134.1.31

Gilpin, A. T. (2009). Mechanisms for overcoming reality status biases. Unpublished doctoral dissertation, University of Texas, Austin.

Hakkarainen, P. \& Bredikyte, M. (2015). How play creates the zone of proximal development. En Robson $\mathrm{S}$, Flannery $\mathrm{S}$. editors. The Routledge International Handbook of Young Children's Thinking and Understanding (p. 31-42) Nueva York: Routledge.

Hirsh-Pasek, K., Golinkoff, R. M., Berk, L. E., \& Singer, D. G. (2009). A mandate for playful learning in preschool. Presenting the evidence. New York; Oxford: Oxford University Press.

Hughes, C. (2013). Executive Function. Development, individual differences, and clinical insights. In Neural Circuit Development and Function in the Healthy and Diseased Brain (Vol. \#volume\#, pp. 429-445). Elsevier Inc. https://doi. org/10.1016/B978-0-12-397267$5.00062-5$

Karpov, Y. V. (2005). The Neo-Vygotskian Approach to Child Development. New York: Cambridge University Press.

Kelly, R. \& Hammond, S. (2011). The relationship between symbolic play and executive function in young children. Australasian journal of early childhood, 36(2), $21-27$.

Kroll, L. (2016). Early childhood curriculum development: the role of play in the 
building self-regulatory capacity in young children. Early child development and care, $1-15$, doi: 10.1080/03004430.2016.1223063.

Lewis, V., \& Boucher, J. (1997). The test of pretend play, ToPP. London: The Psychological Corporation.

Lillard, A., Lerner, M., Hopkins, E., Dore, R., Smith, E. \& Palmquist, C. (2013). The impact of pretend play on children's development: a review of the evidence. Psychological bulletin, 139(1), p. $1-34$.

Lipina, S. \& Segretin, M. (2015). 6000 días más: evidencia neurocientífica acerca del impacto de la pobreza infantil. Psicología Educativa, 21, 107 - 116. Doi:10.1016/j. pse.2015.08.003.

Miyake, a, Friedman, N. P., Emerson, M. J., Witzki, a H., Howerter, a, \& Wager, T. D. (2000). The unity and diversity of executive functions and their contributions to complex "Frontal Lobe" tasks: a latent variable analysis. Cognitive Psychology, 41(1), 49-100. https:// doi.org/10.1006/cogp.1999.0734

Moriguchi, Y. (2014). The early development of executive function and its relation to social interaction: a brief review. Frontiers in Psychology, 5(April), $388 . \quad$ https://doi.org/10.3389/ fpsyg. 2014.00388

Nicolopoulou, A. (1993). Play, cognitive development, and the social world: Piaget, Vygotsky and Beyond. Human development, 36(1), 1 - 23. Nicolopoulou, A. \& Ilgaz, H. (2013). What do we know about pretend play and narrative development? A response to Lillard, Lerner, Hopkins, Dore, Smith and Palmquist on "The impact of pretend play on children's development: a review of the evidence". American journal of play, 6(1), $55-81$.

Nicolopoulou, A., Schnabel Cortina, K., Ilgaz, H., Brockmeyer Cates, C. \& de Sá, A. B. (2015). Using a narrative-and play-based activity to promote low-income preschoolers' oral language, emergent literacy and social competence. Early childhood research quarterly, $147-162$. Doi:10.1016/j.ecresq.2015.01.006

Nigg, J. (2016). Annual research review: on the relations among self-regulation, self-control, executive functioning, effortful control, cognitive control, impulsivity, risk-taking, and inhibition for developmental psychopathology. Journal of child psychology and psychiatry, $1-23$. Doi:10.1111/jcpp.12675.

Overton, W. F., \& Jackson, J. P. (1973). The representation of imagined objects in action sequences: A developmental study. ChildDevelopment, 44, 309314.

Paley, V. G. (1990). The boy who would be a helicopter: The uses of storytelling in theclassroom. Cambridge, MA: Harvard University Press.

Singer, D. G., \& Singer, J. L. (1981). Television, imagination, and aggression: A study of preschoolers. Hillsdale, NJ: Lawrence Erlbaum.

Slot, P., Mulder, H., Verhagen, J. \& Leseman, P. (2017). Preschoolers' cognitive and emotional self-regulation in pretend play: relations with 
executive functions and quality of play. Infant and child development, 1 - 21. Doi:10.1002/icd.2038.

Smilansky, S., \& Shefatya, L. (1990). Facilitating play: A medium for promoting cognitive, socioemotional and academic development in young children. Gaithersburg, MD: Psychosocial and Educational Publications.

Smirnova, E. \& Gudareva, O. (2015). Play and intentionality among today's preschoolers. Journal of Russian and East European Psychology, 52(4), 1 - 20. Doi:10.1080/106104 05.2015.1184891.

Smith, P. (2010). Pretend play: theories and functions. En Children and play. United Kingdom: John Wiley \& Sons, Ltd, Publication

Taylor, M., \& Carlson, S. M. (1997). The relation between individual differences in fantasy and theory of mind. Child Development, 68, 436-455.

Thibodeau, R., Gilpin, A., Brown, M. \& Meyer, B. (2016). The effects of fantastical pretend-play on the development of executive functions: An intervention study. Journal of experimental child psychology, $120-138$. Doi:10.1016/j.jecp.2016.01.001

Vandenplas-Holper, C., \& Deleau, M. (2004). L'observation du Jeu de Faire-Semblant, OJFS. Partial manuscript. Louvain-la-Neuve: Catholic University of Louvain.

Vieillevoye, S. \& Nader-Grosbois, N. (2008).
Self - regulation during pretend play in children with intellectual disability and in normally developing children. Research in developmental disabilities, 29, 256 - 272. Doi:10.1016/j. ridd.2007.05.003

Vygotsky, L. S. (1996). Obras escogidas IV: Psicología infantil. Madrid: Visor.

Vygotsky, L. S. (2000). El desarrollo de los procesos psicológicos superiores. Barcelona: Crítica

Vygotsky, L. S. (2005). Apendix. Journal of Russian and East European psychology, 43(2), 90 - 97.

Whitebread, D. \& Basilio, M. (2012). Emergencia y desarrollo temprano de la autorregulación en niños preescolares. Profesorado, 16(1), $15-34$.

Zaporozhets, A. V. (1980) La importancia de las primeras etapas de la niñez en la formación de la personalidad infantil. Infancia y Aprendizaje, 3(11), $69-75$.

Zelazo, P. D., Müller, U., Frye, D., Marcovitch, S., Argitis, G., Boseovski, J., ... Carlson, S. M. (2003). The Development of Executive Function in Early Childhood, 68(3).

Zelazo, P., Hongwanishkul, D., Happaney, K. \& Lee, W. (2005). Assessment of hot and cool executive function in young children: age-related changes and individual differences. Developmental neuropsychology, 28(2), 617-644. 